\title{
How to Look for Supersymmetry under the LHC Lamppost
}

\author{
Partha Konar, Konstantin T. Matchev, Myeonghun Park, and Gaurab K. Sarangi \\ Physics Department, University of Florida, Gainesville, Florida 32611, USA
}

(Received 17 August 2010; published 22 November 2010)

\begin{abstract}
We apply a model-independent, agnostic approach to the collider phenomenology of supersymmetry, in which all mass parameters are taken as free inputs at the weak scale. We consider the gauginos, Higgsinos, and the first two generations of sleptons and squarks, and analyze all possible mass hierarchies among them $(4 \times 8 !=161280$ in total $)$ in which the lightest superpartner is neutral, leading to missing energy. In each case, we identify the full set of the dominant decay chains originating from the lightest colored superpartner. Our exhaustive search reveals several quite dramatic yet unexplored multilepton signatures with up to 8 isolated leptons (plus possibly up to 2 massive gauge or Higgs bosons) in the final state. Such events are spectacular, background-free for all practical purposes, and may lead to a discovery in the very early stage $\left(\sim 10 \mathrm{pb}^{-1}\right)$ of LHC operations at $7 \mathrm{TeV}$.
\end{abstract}

DOI: 10.1103/PhysRevLett.105.221801

PACS numbers: 14.80.Ly, 12.60.Jv, 13.85.Rm

The ramping operations at the CERN Large Hadron Collider (LHC) have begun the long awaited and historic exploration of the $\mathrm{TeV}$ scale, where new physics beyond the standard model (SM) is expected to emerge. Among the multitude of new scenarios, low energy supersymmetry (SUSY) has long been the primary target of the LHC experiments, not just because it is well motivated theoretically [1], but also because its generic discovery signatures cover a much wider class of models [2].

By itself, SUSY is very predictive, as it fixes the spins and couplings of the new particles (the superpartners). Unfortunately, this is not sufficient to pin down its precise collider discovery signatures, as the latter crucially depend on the SUSY mass spectrum, which is in turn determined by the mechanism of supersymmetry breaking. Alas, almost 40 years of model building effort since the discovery of supersymmetry have failed to produce a single, universally accepted model of SUSY breaking.

Given one's utter ignorance about the expected pattern of SUSY masses, in this letter we adopt a most conservative, agnostic approach, where the masses of all superpartners are treated as free inputs at the weak scale. We shall then consider all possible hierarchical patterns among them, and identify the set of dominant (in the sense defined below) collider signatures in each case. In our quest for interesting models, we shall be guided by experimental pragmatism instead of theoretical prejudice.

The purpose of this study is twofold. First, most previous collider studies of SUSY have been performed for specific SUSY benchmark points, typically chosen within some minimal model such as "minimal supergravity" (MSUGRA) [3]. We will therefore be interested in uncovering new types of signatures which may have been missed in the standard benchmark approach. Secondly, we shall focus our search on signatures with a high number of isolated leptons, which constitute the proverbial "smoking gun" for new physics. For example, the inclusive trilepton channel is already recognized as "the golden mode" for an early SUSY discovery at hadron colliders. One of our main results here will be the identification of a number of new SUSY mass patterns whose dominant signatures have up to eight leptons in the final state.

Our setup is as follows. We take the usual superpartner content of the minimal supersymmetric standard model (MSSM) listed in Table I. For simplicity, we consider just two degenerate light generations of sfermions. Third generation effects can be trivially incorporated in the discussion [4], and only complicate the bookkeeping. The mass splittings within the $Q, L, H$ and $W$ multiplets arise as electroweak symmetry breaking effects and can be safely ignored for our purposes. Given the 9 input mass parameters in Table I, in general there are $9 !=362880$ possible orderings among them, each leading to a distinct pattern (hierarchy) of sparticle masses. We shall use the shorthand notation from Table I to label each hierarchy, for example, GQUDHLWEB is a model with $M_{G}>M_{Q}>$ $M_{U}>M_{D}>M_{H}>M_{L}>M_{W}>M_{E}>M_{B}$.

Our first goal is to identify the main collider signatures for each hierarchy. Our starting point is the fate and then the nature of the Lightest Supersymmetric Particle (LSP), which we generically denote by $\mathcal{L}$. For our main analysis, we shall assume that $R$ parity is conserved (or very weakly broken), so that $\mathcal{L}$ is stable on the scale of the detector. (We briefly discuss the $R$-parity violating option at the

TABLE I. The set of SUSY particles considered in this analysis, shorthand notation for each multiplet, and the corresponding soft SUSY breaking mass parameter.

\begin{tabular}{lccccccccc}
\hline \hline$\tilde{u}_{L}, \tilde{d}_{L}$ & $\tilde{u}_{R}$ & $\tilde{d}_{R}$ & $\tilde{e}_{L}, \tilde{\nu}_{L}$ & $\tilde{e}_{R}$ & $\tilde{h}^{ \pm}, \tilde{h}_{u}^{0}, \tilde{h}_{d}^{0}$ & $\tilde{b}^{0}$ & $\tilde{w}^{ \pm}, \tilde{w}^{0}$ & $\tilde{g}$ \\
\hline$Q$ & $U$ & $D$ & $L$ & $E$ & $H$ & $B$ & $W$ & $G$ \\
$M_{Q}$ & $M_{U}$ & $M_{D}$ & $M_{L}$ & $M_{E}$ & $M_{H}$ & $M_{B}$ & $M_{W}$ & $M_{G}$ \\
\hline \hline
\end{tabular}


end.) Then, the original 9! model hierarchies can be classified into the following three categories:

I. CHAMPs. - In the $8 !=40320$ cases with $\mathcal{L}=E$, the LSP is an electrically charged, color-neutral particle (the right-handed slepton $\tilde{e}_{R}$ ). The corresponding generic collider signature is a long-lived charged massive particle (CHAMP) [5], regardless of the particular ordering of the heavier sparticles.

II. $R$ hadrons. - In $4 \times 8 !=161280$ of the remaining hierarchies $\mathcal{L} \in\{Q, U, D, G\}$, the LSP is colored, and the generic searches for stable $R$-hadrons apply [6]. Again the ordering of the heavier particles is unimportant.

III. Missing transverse energy.-In the remaining $4 \times 8$ ! $=161280$ cases $\mathcal{L} \in\{L, B, W, H\}$ and the LSP is a weakly-interacting, electrically neutral particle. Its production will lead to missing transverse energy $\left(\mathscr{E}_{T}\right)$ in the detector. Now, however, the signatures crucially depend on the ordering of the heavier particles, since it is not feasible to look for $\mathbb{E}_{T}$ inclusively. Our goal here is to fully classify these 161280 models according to their collider phenomenology. Unlike previous general approaches [7], which employed scans of the multidimensional SUSY parameter space, here we would like to avoid scanning, keeping the discussion simple and qualitative.

Both of the currently operating high energy colliders (the Tevatron at Fermilab and the LHC at CERN) are hadron machines, at which the total production is expected to be dominated by the strong production of colored superpartners. Correspondingly, the starting point for our classification will be the nature of the lightest colored superpartner (LCP), denoted by $\mathcal{C}$. Then, each of the 161280 missing energy hierarchies at hand can be represented by a particular ordering

$$
x \ldots x \mathcal{C} y \ldots y \mathcal{L},
$$

where the $x$ 's stand for inconsequential entries, $\mathcal{C} \in$ $\{G, Q, U, D\}, y \in\{L, B, W, H, E\}$ and $\mathcal{L} \in\{L, B, W, H\}$. The dominant collider signature for each model hierarchy (1) will be determined by the inclusive pair production of $\mathcal{C}$ and its dominant subsequent decays.

Our key idea here is that once a given hierarchy (1) is assumed, the dominant decay modes of $\mathcal{C}$ are uniquely determined, since supersymmetry predicts all superpartner couplings. In our analysis, we shall assume that there are no accidental phase space suppressions due to any two mass parameters from Table I being very close. This assumption also guarantees that the chargino and neutralino mixing angles are small and the mass eigenstates are roughly aligned with the interaction eigenstates. One can then use the simple chart in Fig. 1 to identify the dominant (i.e., least suppressed) decay modes of $\mathcal{C}$, which we label by the number of leptons $n_{\ell}$ (blue lines), number of jets $n_{j}$ (red lines) and number of massive bosons $n_{v}$ (green lines) encountered along the way. Solid lines in the figure correspond to two-body decays which do not suffer from any (chargino or neutralino) mixing angle suppression (MAS); dashed lines indicate either two-body decays with MAS or

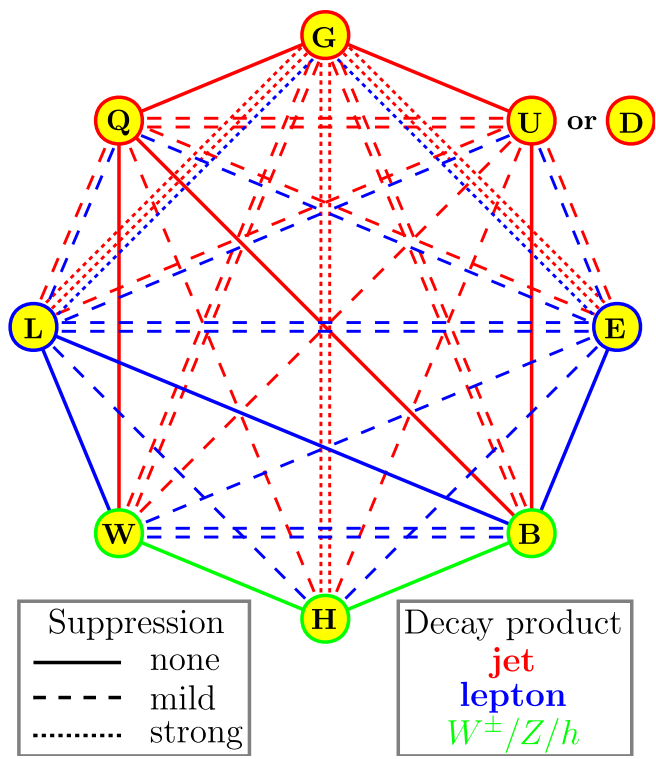

FIG. 1 (color online). Graphical representation of the allowed transitions between the SUSY states from Table I. One (two, three) parallel lines represent two- (three-, four-) body decays. The relative suppression of each decay mode is indicated by the line type. The identity of the resulting SM decay products is denoted by the line color: red for a jet $j$, blue for a lepton $\ell$ and green for a massive boson $v \equiv\left\{W^{ \pm}, Z, h\right\}$ (which may be either on-shell or off-shell).

three-body decays with no MAS; and finally, the dotted lines stand for either three-body decays with MAS or fourbody decays with no MAS. We then count the number of mass hierarchies (1) which exhibit a dominant decay channel for $\mathcal{C}$ with a given set of $\left(n_{\ell}, n_{v}, n_{j}\right)$, and show the result in Table II. At times, there can be several dominant decay modes of $\mathcal{C}$. For example, consider $x x x x Q W B L H$. One can get $\left(n_{\ell}, n_{v}, n_{j}\right)=(0,1,1)$ in two ways: $Q \rightarrow W \rightarrow H$ or $Q \rightarrow B \rightarrow H$. It is also possible to have $\left(n_{\ell}, n_{v}, n_{j}\right)=$ $(2,0,1)$ in two different ways: $Q \rightarrow W \rightarrow L \rightarrow H$ or $Q \rightarrow$ $B \rightarrow L \rightarrow H$. Therefore, $x \times x x Q W B L H$ contributes one entry to each of the two boxes $\left(n_{\ell}, n_{v}, n_{j}\right)=(0,1,1)$ and $\left(n_{\ell}, n_{v}, n_{j}\right)=(2,0,1)$ in Table II. As a result, the total number of entries (203 184) in Table II is larger than the total number of hierarchies (161280).

Table II leads to some interesting conclusions. For example, we see that the purely hadronic signatures of $n_{j}$ jets

TABLE II. Number of hierarchies for the various dominant decay modes of the LCP $\mathcal{C}$.

\begin{tabular}{ccccccc}
\hline \hline & \multicolumn{2}{c}{$n_{v}=0$} & \multicolumn{2}{c}{$n_{v}=1$} & \multicolumn{2}{c}{$n_{v}=2$} \\
$n_{\ell}$ & $n_{j}=1$ & $n_{j}=2$ & $n_{j}=1$ & $n_{j}=2$ & $n_{j}=1$ & $n_{j}=2$ \\
\hline 0 & 79296 & 26880 & 12768 & 3360 & 1344 & 672 \\
1 & 30240 & 10080 & 1824 & 480 & 192 & 96 \\
2 & 19770 & 6030 & 1500 & 180 & 0 & 0 \\
3 & 4656 & 1296 & 312 & 72 & 6 & 6 \\
4 & 1656 & 396 & 66 & 6 & 0 & 0 \\
\hline \hline
\end{tabular}


and $\mathscr{E}_{T}$ alone cover a very large fraction $(\sim 65 \%)$ of all possible SUSY hierarchies with a neutral LSP. A sizable fraction of models can also be explored via the standard searches for signatures with one, two, or three leptons. Keep in mind that the LCP's are produced in pairs, so the collider signature is obtained by doubling the number of leptons and jets displayed in the table and is given by $2 n_{\ell}+2 n_{v}+2 n_{j}+\mathbb{E}_{T}$. Perhaps the most important result from Table II is that there is a non-negligible fraction of SUSY models $(\sim 1 \%)$ in which one of the dominant LCP decay modes yields 4 isolated leptons, and the corresponding collider signature is 8 leptons plus $\mathscr{E}_{T}$.

We now repeat the same analysis, only this time from the set of all dominant decay modes of $\mathcal{C}$, we select the one with the largest $n_{\ell}$, and in case of a tie for $n_{\ell}$, we pick the chain with the larger $n_{v}$. We refer to such decay modes of the LCP as "maximally leptonic". The new tally is displayed in Table III, where now the sum of all entries equals the total number of signatures 161280.

It is instructive to apply our general formalism to the familiar MSUGRA model, where the soft SUSY mass parameters of Table I have common values $\left(M_{0}\right.$ for the scalar superpartners and $M_{1 / 2}$ for the gauginos) at the grand unification scale. First, one may ask how many of the 161280 missing energy hierarchies are actually represented in MSUGRA. The answer is provided in Fig. 2, in which we divide the usual $\left(M_{0}, M_{1 / 2}\right)$ plane into disjoint color-coded areas, according to the observed mass pattern (1). The allowed region contains only 47 different hierarchies (some areas are too small to be readily noticeable with the naked eye). Comparing this to the total number of 161280 possibilities, one gets an idea of the limitations of MSUGRA as a benchmark scenario.

Figure 2 also illustrates the extent to which the MSUGRA model is able to cover the generic SUSY signature space. The black solid lines in Fig. 2 divide the allowed portion of the $\left(M_{0}, M_{1 / 2}\right)$ plane into disjoint regions, classified according to the maximally leptonic $\mathcal{C}$ decay mode, labeled by $\left(n_{\ell}, n_{v}, n_{j}\right)$ and determined simply [8] from Fig. 1. We find that MSUGRA exhibits only 4 out of the 26 possibilities found in Table III, namely $\left(n_{\ell}, n_{v}, n_{j}\right)=(0,0,1),(2,0,2),(0,2,2)$, and $(0,1,2)$. Of course, Fig. 2 only displays dominant LCP decay modes. Some parameter points in MSUGRA may contain other, longer decay chains with a higher number of leptons,

TABLE III. Number of hierarchies for the maximally leptonic decay modes of the LCP $\mathcal{C}$.

\begin{tabular}{ccccccc}
\hline \hline & \multicolumn{2}{c}{$n_{v}=0$} & \multicolumn{2}{c}{$n_{v}=1$} & \multicolumn{2}{c}{$n_{v}=2$} \\
$n_{\ell}$ & $n_{j}=1$ & $n_{j}=2$ & $n_{j}=1$ & $n_{j}=2$ & $n_{j}=1$ & $n_{j}=2$ \\
\hline 0 & 61488 & 21168 & 8310 & 2550 & 780 & 420 \\
1 & 24150 & 8310 & 1278 & 378 & 132 & 72 \\
2 & 17190 & 5550 & 1230 & 150 & 0 & 0 \\
3 & 4362 & 1242 & 312 & 72 & 6 & 6 \\
4 & 1656 & 396 & 66 & 6 & 0 & 0 \\
\hline \hline
\end{tabular}

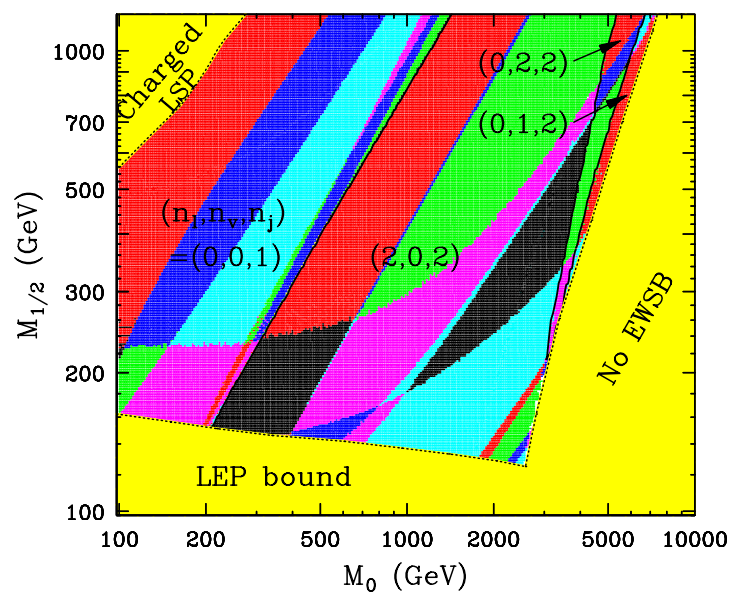

FIG. 2 (color online). A slice through the MSUGRA parameter space for fixed $A_{0}=0, \tan \beta=10$ and $\mu>0$. Yellow shaded regions are ruled out by direct searches at LEP or by requiring a neutral LSP. The remaining area is color-coded according to the sparticle hierarchy type (1). The solid black lines delineate regions with the same maximally leptonic $\mathcal{C}$ decay mode.

but those would be subdominant and therefore suppressed by branching fractions. For example, the hierarchy $x x x U H L W E B$ has a 4-lepton chain $\tilde{u}_{R} \stackrel{j}{\rightarrow} \tilde{h}^{0} \stackrel{\ell}{\rightarrow} \tilde{e}_{L} \stackrel{\ell}{\rightarrow} \tilde{w}^{0} \stackrel{\ell}{\rightarrow} \tilde{e}_{R} \stackrel{\ell}{\rightarrow} \tilde{b}^{0}$, but it has two bottlenecks at $\tilde{u}_{R} \stackrel{j}{\rightarrow} \tilde{h}^{0}$ and $\tilde{h}^{0} \stackrel{\ell}{\rightarrow} \tilde{e}_{L}$; see Fig. 1 .

In the rest of this Letter we study in detail one example [9] of a maximally leptonic decay chain with 4 leptons, where the corresponding collider signal is 8 isolated leptons plus jets plus missing energy. For concreteness, consider the hierarchy $x x G Q W L B E H$ at several different study points, defined in Table IV and chosen "under the lamppost", i.e., to maximize the 8-lepton signal rate for a given value of $M_{Q}$. For this hierarchy, 8 lepton events arise from the inclusive pair production of left-handed squarks $\tilde{u}_{L}, \tilde{d}_{L}$, followed by

$$
\tilde{u}_{L}, \tilde{d}_{L} \stackrel{j}{\rightarrow} \tilde{w}^{0} \stackrel{\ell}{\rightarrow} \tilde{\ell}_{L} \stackrel{\ell}{\rightarrow} \tilde{b}^{0} \stackrel{\ell}{\rightarrow} \tilde{\ell}_{R} \stackrel{\ell}{\rightarrow} \tilde{h}_{u}^{0}, \tilde{h}_{d}^{0} .
$$

Ignoring phase space suppression factors, third generation effects and the masses of the $W^{ \pm}, Z$ and $h$, it is easy to

TABLE IV. Input soft SUSY mass parameters (in $\mathrm{GeV}$ ) for the $x x G Q W L B E H$ study points used for Figs. 3 and 4.

\begin{tabular}{lllllll}
\hline \hline$M_{G}$ & $M_{Q}$ & $M_{W}$ & $M_{L}$ & $M_{B}$ & $M_{E}$ & $M_{H}$ \\
\hline 400 & 300 & 220 & 190 & 130 & 130 & 130 \\
450 & 350 & 280 & 190 & 120 & 120 & 120 \\
500 & 400 & 280 & 190 & 120 & 120 & 120 \\
550 & 450 & 310 & 200 & 120 & 120 & 120 \\
600 & 500 & 350 & 210 & 130 & 120 & 120 \\
700 & 600 & 420 & 230 & 150 & 130 & 120 \\
800 & 700 & 480 & 250 & 160 & 130 & 120 \\
900 & 800 & 500 & 250 & 170 & 130 & 120 \\
1000 & 900 & 510 & 250 & 170 & 130 & 120 \\
\hline \hline
\end{tabular}




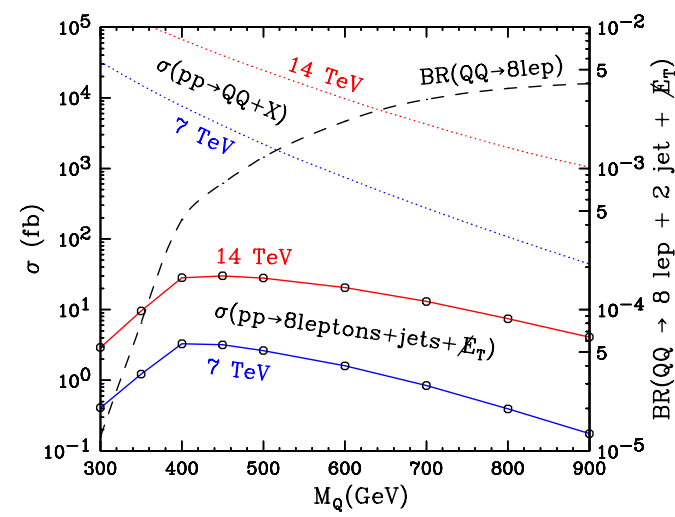

FIG. 3 (color online). Inclusive cross sections (in $\mathrm{fb}$ ) at the LHC for 8 lepton events from the exclusive chain in (2) for the study points from Table IV (solid lines); inclusive cross sections for left-handed squark pair production (dotted); and cumulative branching fraction of the 8 lepton final state (dashed, read on the right).

estimate the individual branching ratios for this chain as follows [4]: $B R\left(\tilde{g} \rightarrow \tilde{q}_{L}+j\right)=1 ; B R\left(\tilde{q}_{L} \rightarrow \tilde{w}^{0}+j\right) \simeq \frac{1}{3}$; $B R\left(\tilde{w}^{0} \rightarrow \tilde{\ell}_{L}^{ \pm}+\ell^{\mp}\right) \simeq \frac{1}{3} ; B R\left(\tilde{\ell}_{L}^{ \pm} \rightarrow \tilde{b}^{0}+\ell^{ \pm}\right)=1 ;$ $B R\left(\tilde{b}^{0} \rightarrow \tilde{\ell}_{R}^{ \pm}+\ell^{\mp}\right) \simeq \frac{4}{5} ; B R\left(\tilde{\ell}_{R}^{ \pm} \rightarrow \tilde{h}_{u / d}^{0}+\ell^{ \pm}\right)=1$. Multiplying these results and squaring, we expect the cumulative branching fraction for 8 lepton events to be around $0.7 \%$. This is confirmed by the dashed line in Fig. 3, which approaches this asymptotic value for large mass splittings (large $M_{Q}$ ). We see that to maximize the overall branching fraction, we must scale the SUSY mass spectrum up. Yet to maximize the production rate, we must scale the spectrum down. An optimum compromise is therefore found for intermediate values of the SUSY masses, as shown in Fig. 3. The study points in Table IV were picked by varying $M_{Q}$, fixing $M_{G}=M_{Q}+$ $100 \mathrm{GeV}$, and choosing the rest of the spectrum from a coarse scan to maximize the 8 lepton rate shown by the solid lines in Fig. 3. These study points were then processed with the PYTHIA [10] event generator and the PGS [11] detector simulator for the case of an LHC at $7 \mathrm{TeV}$ center-of-mass energy and just $1 \mathrm{fb}^{-1}$ of data. Because these events are essentially background-free, we simply count PGS-reconstructed isolated leptons with default $p_{T}$ cuts of $3 \mathrm{GeV}$ for muons and $10 \mathrm{GeV}$ for electrons, and display the result in Fig. 4. Because of the imperfect detector acceptance, we got only one 8 lepton event in $1 \mathrm{fb}^{-1}$. Nevertheless, for $M_{Q} \lesssim 500 \mathrm{GeV}$ typically there are a handful of 7 lepton events and hundreds of 4 lepton events, which are already very clean. If we require five such clean events for discovery, the region $M_{Q} \lesssim 500 \mathrm{GeV}$ can be probed with as little as $10 \mathrm{pb}^{-1}$ of data.

We note in passing that one may expect even more leptons if $R$ parity is violated and the LSP decays promptly to SM particles. For example, lepton number violation of

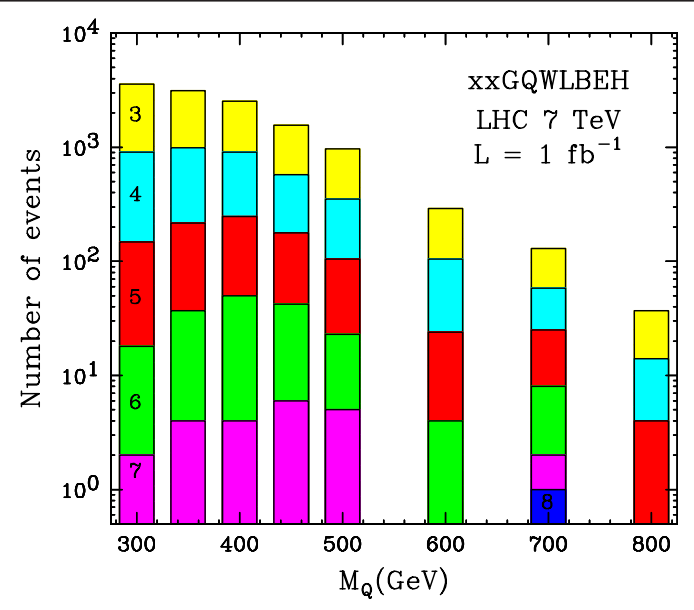

FIG. 4 (color online). (Stacked) number of multilepton events in $1 \mathrm{fb}^{-1}$ of data at a $7 \mathrm{TeV} \mathrm{LHC}$, for the $x x G Q W L B E H$ sparticle hierarchy with the mass spectra shown in Table IV.

the type $L L E$ would lead to $2+2=4$ additional leptons, bringing the maximum lepton total per event to 12 .

In conclusion, we have shown that already with its first $10 \mathrm{pb}^{-1}$ of data the LHC can start probing SUSY, provided one looks in the right place. To this end, it is imperative to search for the model hierarchies that are most likely to be discovered first. We believe this letter outlines the most general strategy to do just that.

Work supported by US DOE grant DE-FG0297ER41029.

[1] D. J. H. Chung et al., Phys. Rep. 407, 1 (2005).

[2] H.-C. Cheng, K. T. Matchev, and M. Schmaltz, Phys. Rev. D 66, 056006 (2002).

[3] M. Battaglia et al., Eur. Phys. J. C 22, 535 (2001).

[4] P. Konar, K. Matchev, M. Park, and G. Sarangi (to be published).

[5] T. Aaltonen et al. (CDF Collaboration), Phys. Rev. Lett. 103, 021802 (2009); V.M. Abazov et al. (D0 Collaboration), Phys. Rev. Lett. 102, 161802 (2009).

[6] A. C. Kraan, J. B. Hansen, and P. Nevski, Eur. Phys. J. C 49, 623 (2007).

[7] See, e.g., D. Feldman, Z. Liu, and P. Nath, Phys. Rev. Lett. 99, 251802 (2007); C.F. Berger et al., J. High Energy Phys. 02 (2009) 023; C. Horn, J. Phys. G 36, 105005 (2009).

[8] The actual result in MSUGRA looks slightly different, due to third generation effects and numerical competition between the $\tilde{w}^{0} \stackrel{\ell \ell}{\rightarrow} b^{0}$ and $\tilde{w}^{0} \stackrel{Z / h}{\rightarrow} b^{0}$ decay modes.

[9] The complete list of all 161280 hierarchies analyzed in this Letter and their signatures will be given in [4].

[10] T. Sjostrand, S. Mrenna, and P.Z. Skands, J. High Energy Phys. 05 (2006) 026.

[11] http://www.physics.ucdavis.edu/ conway/research/ software/pgs/pgs4-general.htm. 